\title{
Erratum to: Lipid-modified azurin of Neisseria meningitidis is a copper protein localized on the outer membrane surface and not regulated by FNR
}

\author{
Manu Deeudom • Wilhemina Huston • \\ James WB Moir
}

Published online: 10 March 2015

(C) Springer International Publishing Switzerland 2015

\section{Erratum to: Antonie van Leeuwenhoek DOI 10.1007/s10482-015-0400-z}

In the original published version, the captions provided for the two supplementary figures were not included in the electronic supplementary material.

Below are the captions to the supplementary figures:

Supplementary Figure 1 Expression of meningococcal Laz protein in E. coli. (A) Periplasmic fraction containing Laz protein was expressed in Laz expressing strain BL21( $\lambda \mathrm{DE} 3)$. Lane $1=$ control, Lane $2=$ Laz-expressing strain. Three different sizes of Laz were found in the polyacrylamide gel electrophoresis done under non-reducing conditions. (B) Purified Laz from DEAE-sepharose anion exchange chromatography. The purified protein was treated with

The online version of the original article can be found under doi:10.1007/s10482-015-0420-8.

M. Deeudom $(\bowtie) \cdot$ J. W. Moir

Department of Biology (Area 10), University of York,

Heslington, York YO10 5YW, UK

e-mail: manu.deeudom@cmu.ac.th

M. Deeudom

Department of Microbiology, Faculty of Medicine,

Chiang Mai University, Chiang Mai 50200, Thailand

W. Huston

Institute of Health and Biomedical Innovation,

Queensland University of Technology, Kelvin Grove,

QLD 4059, Australia $\beta$-mercaptoethanol to break disulphide bond and reduce the aggregation of protein. The soluble Laz protein exhibits molecular mass approximately at 24 $\mathrm{kDa}$ on SDS-PAGE. Lane 1-7 are protein fractions containing Laz.

Supplementary Figure 2 Molecular mass of Laz detected by electrospray mass spectrophotometry. (A) Laz has a molecular mass of 16,983.6 $\pm 1 \mathrm{Da}$ analyzed in acidic conditions. (B) Laz has a molecular mass of $17,045.6 \pm 1 \mathrm{Da}$ analyzed in non-acidic conditions with $20 \%$ methanol solvent. The copper ion has a molecular mass of 63.54 Da corresponding to the difference in mass between the two conditions, indicating that copper is dissociated from Laz under acidic conditions. The major adduct peak with mass of $17,067.6 \pm 1$ Da may be from an association of sodium ion or magnesium ion with a molecular mass of 23.0 Da and 24.3 Da, respectively, and a loss of proton. 